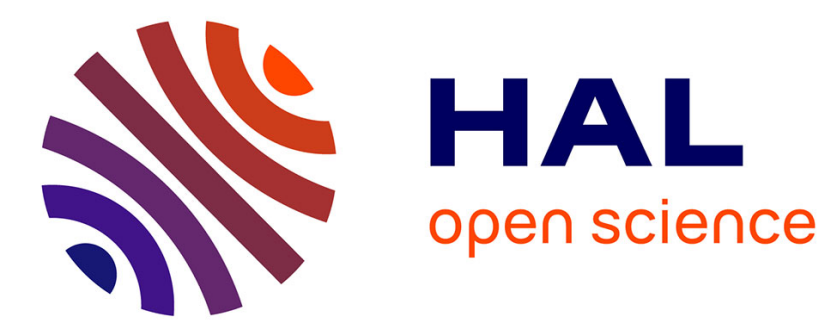

\title{
Comparison of 3-RPR Planar Parallel Manipulators with regard to their Dexterity and Sensitivity to Geometric Uncertainties
}

Nicolas Binaud, Stéphane Caro, Philippe Wenger

\section{- To cite this version:}

Nicolas Binaud, Stéphane Caro, Philippe Wenger. Comparison of 3-RPR Planar Parallel Manipulators with regard to their Dexterity and Sensitivity to Geometric Uncertainties. Meccanica, 2011, 46 (14), pp.75-88. 10.1007/s11012-010-9390-0 . hal-00833524

\section{HAL Id: hal-00833524 https://hal.science/hal-00833524}

Submitted on 13 Jun 2013

HAL is a multi-disciplinary open access archive for the deposit and dissemination of scientific research documents, whether they are published or not. The documents may come from teaching and research institutions in France or abroad, or from public or private research centers.
L'archive ouverte pluridisciplinaire HAL, est destinée au dépôt et à la diffusion de documents scientifiques de niveau recherche, publiés ou non, émanant des établissements d'enseignement et de recherche français ou étrangers, des laboratoires publics ou privés. 


\title{
COMPARISON OF 3-RPR PLANAR PARALLEL MANIPULATORS WITH REGARD TO THEIR KINETOSTATIC PERFORMANCE AND SENSITIVITY TO GEOMETRIC UNCERTAINTIES
}

\author{
Nicolas Binaud, Stéphane Caro, Philippe Wenger \\ Institut de Recherche en Communications et Cybernétique de Nantes \\ UMR CNRS n ${ }^{\circ} 6597$ \\ 1 rue de la Noë, 44321 Nantes, France \\ \{binaud, caro, wenger\}@irccyn.ec-nantes.fr
}

\begin{abstract}
This paper deals with the sensitivity analysis of 3-RPR planar parallel manipulators. First, the manipulators under study as well as their degeneracy conditions are presented. Then, an optimization problem is formulated in order to obtain their maximal regular dexterous workspace. Moreover, the sensitivity coefficients of the pose of the manipulator moving platform to variations in the geometric parameters and in the actuated variables are expressed algebraically. Two aggregate sensitivity indices are determined, one related to the orientation of the manipulator moving platform and another one related to its position. Then, we compare two non-degenerate and two degenerate 3-R $\underline{P} R$ planar parallel manipulators with regard to their dexterity, workspace size and sensitivity. Finally, two actuating modes are compared with regard to their sensitivity.
\end{abstract}

KEYWORDS: Sensitivity analysis, degenerate manipulators, regular dextrous workspace, transmission angle.

\section{Introduction}

Variations in the geometric parameters of PKMs can be either compensated or amplified. For that reason, it is important to analyze the sensitivity of the mechanism performance to variations in its geometric parameters. For instance, Wang et al. [1] studied the effect of manufacturing tolerances on the accuracy of a Stewart platform. Kim et al. [2] used a forward error bound analysis to find the error bound of the end-effector of a Stewart platform when the error bounds of the joints are given, and an inverse error bound analysis to determine those of the joints for the given error bound of the end-effector. Kim and Tsai [3] studied the effect of misalignment of linear actuators of a 3-Degree of
Freedom (DOF) translational parallel manipulator on the motion of its moving platform. Caro et al. [4] developed a tolerance synthesis method for mechanisms based on a robust design approach. Cardou et al. [5] proposed some kinematic-sensitivity indices for dimensionally nonhomogeneous jacobian matrices. Caro et al. [6] proposed two indices to evaluate the sensitivity of the end-effector pose (position + orientation) of Orthoglide 3-axis, a 3-DOF translational PKM, to variations in its design parameters. Besides, they noticed that the better the dexterity, the higher the accuracy of the manipulator. However, Yu et al. [7] claimed that the accuracy of a 3-DOF Planar Parallel Manipulator (PPM) is not necessarily related to its dexterity. Meng et al. [8] proposed a method to analyze the accuracy of parallel manipulators with joint clearances and ended up with a standard convex optimization problem to evaluate the maximal pose error in a prescribed workspace.

Some architectures of planar parallel manipulators are compared with regard to their sensitivity to geometric uncertainties in [9].

This paper deals with the comparison of the sensitivity of two degenerate and two non-degenerate 3-RPR PPMs. Likewise, the sensitivity of two actuating modes of the 3-RPR PPM, namely the 3-RPR PPM and the 3-RㅛR PPM, is analyzed. First, the degeneracy conditions of 3-R $\underline{P} R$ manipulators and the manipulators under study are presented. Then, the formulation of an optimization problem is introduced to obtain the regular dexterous workspace of those manipulators. Finally, a methodology is introduced to analyze and compare the sensitivity of the pose of their moving platforms to variations in their geometric parameters. 


\section{Manipulator Architecture}

Here and throughout this paper, $R, P$ and $\underline{P}$ denote revolute, prismatic and actuated prismatic joints, respectively. Figure. 1 illustrates the architecture of the manipulator under study. It is composed of a base and a moving platform (MP) connected with three legs. Points $A_{1}, A_{2}$ and $A_{3},\left(C_{1}, C_{2}\right.$ and $C_{3}$, respectively) lie at the corners of a triangle, of which point $O$ (point $P$, resp.) is the circumcenter. Each leg is composed of a $R$, a $P$ and a $R$ joints in sequence. The three $\mathrm{P}$ joints are actuated. Accordingly, the manipulator is named 3-R $\underline{P R}$ manipulator.

$\mathcal{F}_{b}$ and $\mathcal{F}_{p}$ are the base and the moving platform frames of the manipulator. In the scope of this paper, $\mathcal{F}_{b}$ and $\mathcal{F}_{p}$ are supposed to be orthogonal. $\mathcal{F}_{b}$ is defined with the orthogonal dihedron $(\overrightarrow{O x}, \overrightarrow{O y})$, point $O$ being its center and $\overrightarrow{O x}$ parallel to segment $A_{1} A_{2}$. Likewise, $\mathcal{F}_{p}$ is defined with the orthogonal dihedron $(\overrightarrow{P X}, \overrightarrow{P Y})$, point $C$ being its center and $\overrightarrow{P X}$ parallel to segment $C_{1} C_{2}$.

The pose of the manipulator MP, i.e., its position and its orientation, is determined by means of the Cartesian coordinates vector $\mathbf{p}=\left[p_{x}, p_{y}\right]^{T}$ of operation point $P$ expressed in frame $\mathcal{F}_{b}$ and angle $\phi$, that is the angle between frames $\mathcal{F}_{b}$ and $\mathcal{F}_{p}$. Finally, the passive joints do not have any stop.

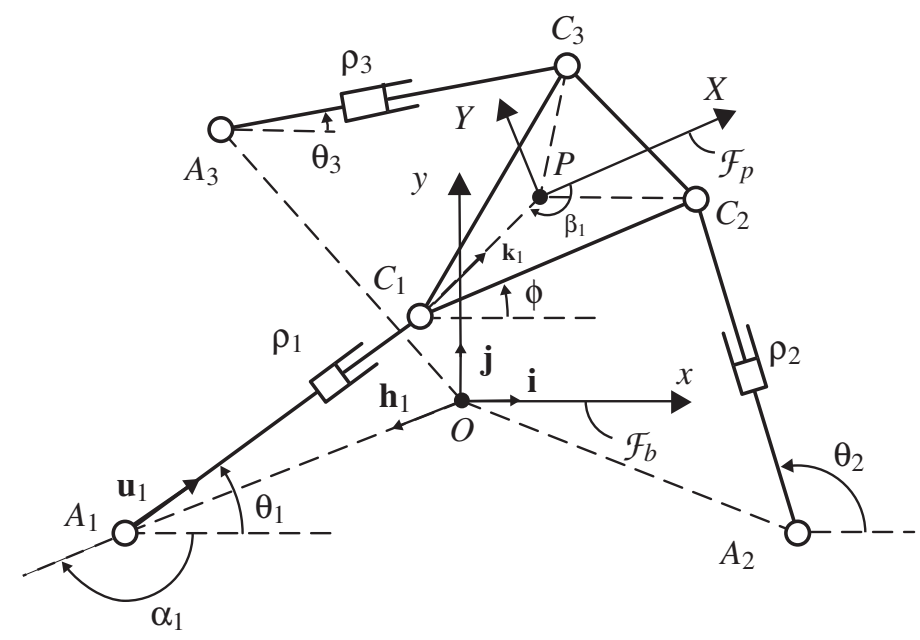

Figure 1. 3-RPR manipulator

\section{Degenerate and Non-Degenerate Manipulators}

In this section, we focus on the sensitivity analysis of two degenerate and two non-degenerate 3-RPR manipulators. First, the degeneracy conditions of such manipulators are given. Then, the architectures of the four manipulators under study are illustrated.

\subsection{Degeneracy condition}

The forward kinematic problem of a parallel manipulator often leads to complex equations and non analytic solutions, even when considering 3-DOF PPMs [10]. For those manipulators, Hunt showed that the forward kinematics admits at most six solutions [11] and some authors proved that their forward kinematics can be reduced to the solution of a sixth-degree characteristic polynomial $[12,13]$.

As shown in [14], [15] and [16], a 3-RPR PPM is said to be degenerate when the degree of its characteristic polynomial becomes smaller than six. Six types of degenerate 3-RPR PPMs exists in the literature, namely,

1. 3-RPR PPMs with two coincident joints;

2. 3-RPR PPMs with similar aligned base and moving platforms;

3. 3-RPR PPMs with nonsimilar aligned base and moving platforms;

4. 3-R $\underline{P} R$ PPMs with similar triangular base and moving platforms;

5. 3-RPR PPMs with the three actuated prismatic joints satisfying a certain relationship;

6. 3-RPR PPMs with congruent base and moving platforms, of which the moving platform is rotated of $180 \mathrm{deg}$ about one of its side.

In the scope of this paper, we focus on the sensitivity analysis of the fourth and the sixth cases. For the fourth case, the forward kinematics is reduced to the solution of two quadratics in cascade. For the sixth case, the forward kinematics degenerates over the whole joint space and is reduced to the solution of a third-degree polynomial and a quadratic in sequence.

\subsection{Manipulators under study}

Figures 2(a)-(d) illustrate the four manipulators under study, named $M_{1}, M_{2}, M_{3}$ and $M_{4}$, respectively. $M_{1}$ and $M_{2}$ are nondegenerate manipulators while $M_{3}$ and $M_{4}$ are degenerate manipulators. From Fig. 2(a), the base and moving platforms of $M_{1}$ are equilateral. From Fig. 2(b), the base and moving platforms of $M_{2}$ are identical but in a different geometric configuration for an orientation $\phi=0 . M_{3}$ and $M_{4}$ illustrate the fourth and the sixth degeneracy cases presented in Sec. 3.1. It is noteworthy that the base and moving platforms of $M_{2}, M_{3}$ and $M_{4}$ have the same circumscribed circle, its radius being equal to $\sqrt{2} / 2$. As far as $M_{1}$ is concerned, the circumscribed circle of its moving platform is two times smaller than the one of the base platform.

To compare the sensitivity of these PPMs, the geometric parameters have to be normalized. Therefore, let $R_{1}$ and $R_{2}$ be the radii of the base and moving platforms of the PPM. In order to come up with finite values, $R_{1}$ and $R_{2}$ are normalized as 


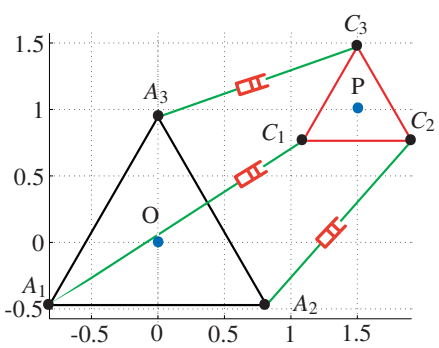

(a) $M_{1}$

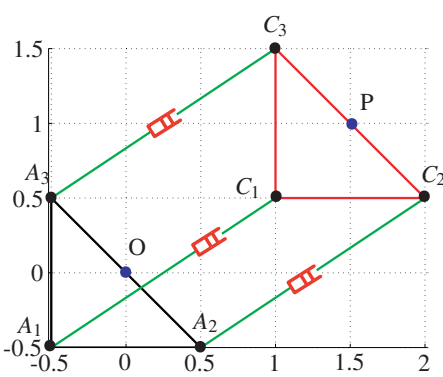

(c) $M_{3}$

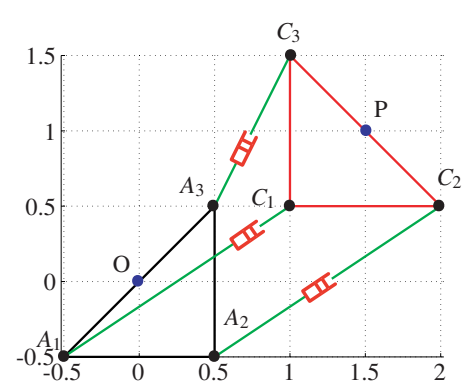

(b) $M_{2}$

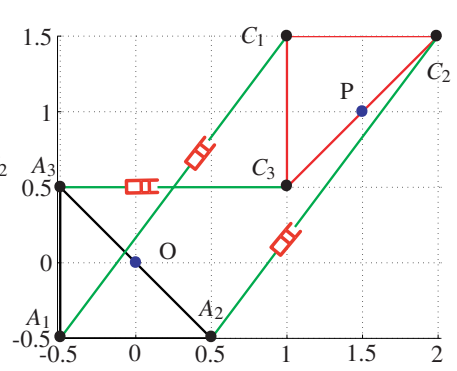

(d) $M_{4}$
Figure 2. The four $3-\mathrm{R} \underline{\mathrm{P}} \mathrm{R}$ manipulators under study with $\phi=0$ and $\mathbf{p}=[1,1.5]^{T}:$ (a)-(b) non-degenerate manipulators, (c)-(d) degenerate manipulators

in [17-19]. For that matter, let $N_{f}$ be a normalizing factor:

$$
N_{f}=\left(R_{1}+R_{2}\right) / 2
$$

and

$$
r_{m}=R_{m} / N_{f}, \quad m=1,2
$$

Therefore,

$$
r_{1}+r_{2}=2
$$

From Eqs.(2) and (3), we can notice that:

$$
r_{1} \in[0,2], r_{2} \in[0,2]
$$

As the former two-dimensional infinite space corresponding to geometric parameters $R_{1}$ and $R_{2}$ is reduced to a one-dimensional finite space defined with Eq.(3), the workspace analysis of the 3-RPR manipulator under study turns out to be easier.

\section{Regular Dexterous Workspace}

In order to compare the sensitivity of the foregoing manipulators, we first define their Regular Dexterous Workspace (RDW). Then, the sensitivity of $M_{1}, M_{2}, M_{3}$ and $M_{4}$ can be evaluated throughout their RDW and compared. The RDW of a manipulator is a regular-shaped part of its workspace with good and homogeneous kinetostatic performance. The shape of the RDW is up to the designer. It may be a cube, a parallelepiped, a cylinder or another regular shape. A good shape fits to the singular surfaces.

The kinetostatic performance of a manipulator is usually characterized by the conditioning number of its kinematic Jacobian matrix [20,21]. From [22,23], the transmission angle of a 3-DOF PPM can be also used to evaluate its kinetostatic performance. Here, we prefer to use the transmission angle as a kinetostatic performance index as it does not require the normalization of the kinematic Jacobian matrix. On the contrary, the kinematic Jacobian matrix of 3-DOF PPM has to be normalized by means of a normalizing length in order its conditioning number to make sense [24].

\subsection{Transmission angle}

The transmission angle $\psi_{i}$ associated with the $i$ th leg is defined as the angle between force vector $\mathbf{F c}_{i}$ and translational velocity vector $\mathbf{V} \mathbf{c}_{i}$ at point $C_{i}$ as illustrated in Fig. 3.

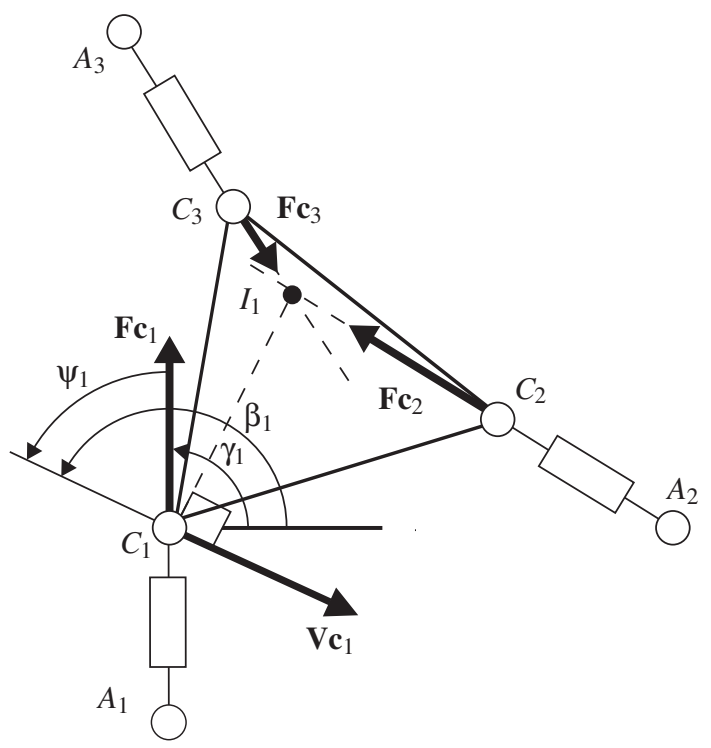

Figure 3. Transmission angle of the 3-R $\underline{P} R$ PPMs

The direction of force $\mathbf{F} \mathbf{c}_{i}$ is the direction of $\operatorname{leg} A_{i} C_{i}$, namely,

$$
\gamma_{i}=\arctan \left(\frac{y_{C_{i}}-y_{A_{i}}}{x_{C_{i}}-x_{A_{i}}}\right), i=1,2,3
$$

The instantaneous centre of rotation depends on the leg under 
study. For example, instantaneous centre of rotation $I_{1}$ associated with leg 1 is the intersecting point of forces $\mathbf{F} \mathbf{c}_{2}$ anf $\mathbf{F} \mathbf{c}_{3}$.

Table 1 gives the Cartesian coordinates of instantaneous centre of rotation $I_{i}$ associated with the $i$ th leg of the 3-R $\underline{P}$ PPM, expressed in frame $\mathcal{F}_{b}$, with $b_{i}=y_{C_{i}}-x_{C_{i}} \tan \gamma_{i}, i=1,2,3$.

The direction of $\mathbf{V} \mathbf{c}_{i}$ is defined as,

$$
\beta_{i}=\arctan \left(\frac{y_{C_{i}}-y_{I_{i}}}{x_{C_{i}}-x_{I_{i}}}\right)+\frac{\pi}{2}, i=1,2,3
$$

Accordingly,

$$
\psi_{i}=\left|\gamma_{i}-\beta_{i}\right|, i=1,2,3
$$

Finally, the transmission angle $\psi$ of the overall mechanism is defined as,

$$
\psi=\max \left(\psi_{i}\right), i=1,2,3
$$

and the smaller $\psi$, the better the force transmission of the mechanism.

\subsection{RDW determination}

In the scope of this study, the RDW of the PPM is supposed to be a cylinder of $\phi$-axis with good kinetostatic performance, i.e., the transmission angle $\psi$ is smaller than $75^{\circ}$ throughout the cylinder. In order to obtain such a RDW, we can solve the following optimization problem:

$$
P b \mid \begin{aligned}
& \min _{\mathbf{x}} 1 / R \\
& \text { s.t. } \quad \Delta \phi \geq 30^{\circ} \\
&
\end{aligned}
$$

$R$ being the radius of the cylinder and $\Delta \phi$ the orientation range of the MP of the manipulator within its RDW. Here, $\Delta \phi$ is equal to $30^{\circ}$ arbitrarily. This optimization problem has five decision variables:

$$
\mathbf{x}=\left[\begin{array}{lllll}
R & I_{x} & I_{y} & \phi_{\min } & \phi_{\max }
\end{array}\right]
$$

$I_{x}$ and $I_{y}$ being the Cartesian coordinates of the center of the cylinder, $\phi_{\min }$ and $\phi_{\max }$ being the lower and upper bounds of $\phi$ angle $\left(\Delta \phi=\phi_{\max }-\phi_{\min }\right)$.

This optimization problem is solved by means of a Tabu search Hooke and Jeeves algorithm [25]. As a result, the RDW of the manipulator is completely defined by means of the decision variables corresponding to the global minimum ${ }^{1}$.

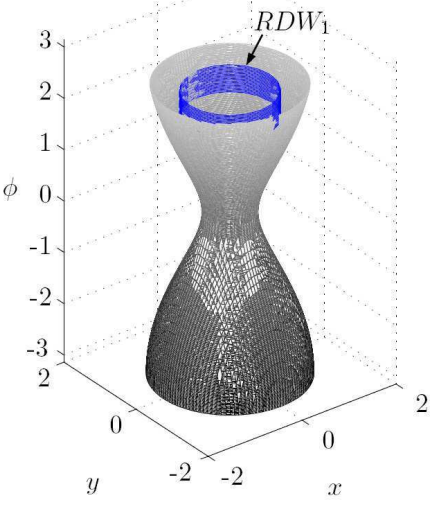

(a) $M_{1}$

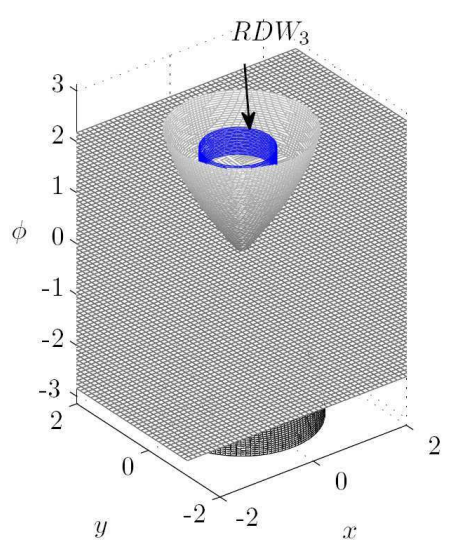

(c) $M_{3}$

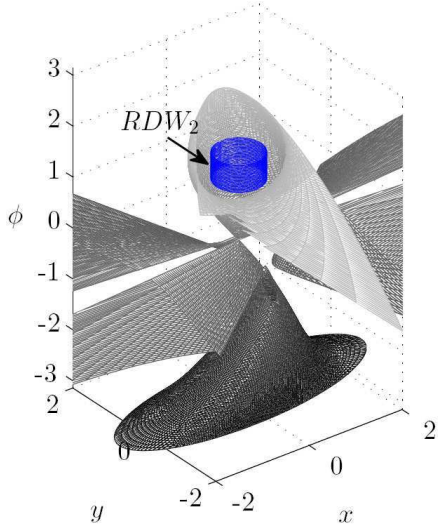

(b) $M_{2}$

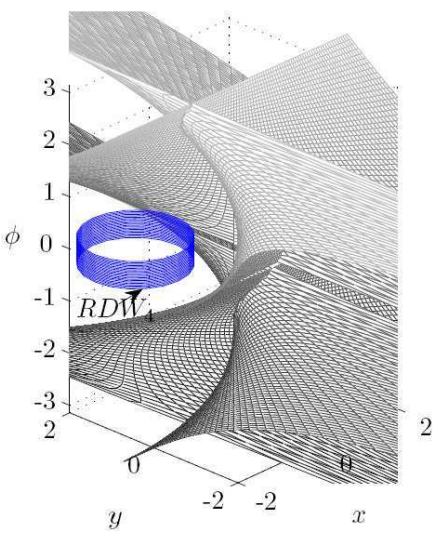

(d) $M_{4}$
Figure 4. Maximal Regular Dexterous Workspace

Table 2. RDW radius of $M_{1}, M_{2}, M_{3}$ and $M_{4}$

\begin{tabular}{cccc}
\hline \hline$R_{1}$ & $R_{2}$ & $R_{3}$ & $R_{4}$ \\
1.21 & 0.62 & 0.75 & 2.69 \\
\hline \hline
\end{tabular}

Figures 4-(d) illustrate the workspace, the singularities and the maximal RDW of $M_{1}, M_{2}, M_{3}$ and $M_{4}$. Their radii are given in Table 2. We can notice that $M_{4}$ has the biggest RDW and $M_{2}$ the smallest one.

\section{Sensitivity Analysis}

In this section, the sensitivity of $M_{1}, M_{2}, M_{3}$ and $M_{4}$ is evaluated throughout their RDW for a matter of comparison. The

\footnotetext{
${ }^{1}$ The solution obtained with a Tabu search Hooke and Jeeves algorithm will not be necessarily the global optimum. However, it will provide a solution that is close to the global one and satisfactory in the framework of this research work.
} 
Table 1. Cartesian coordinates of instantaneous centres of rotation

\begin{tabular}{c|ccc}
\hline \hline & $I_{1}$ & $I_{2}$ & $I_{3}$ \\
\hline$x_{I_{i}}$ & $\frac{b_{3}-b_{2}}{\tan \left(\gamma_{2}\right)-\tan \left(\gamma_{3}\right)}$ & $\frac{b_{1}-b_{3}}{\tan \left(\gamma_{3}\right)-\tan \left(\gamma_{1}\right)}$ & $\frac{b_{2}-b_{1}}{\tan \left(\gamma_{1}\right)-\tan \left(\gamma_{2}\right)}$ \\
\hline$y_{I_{i}}$ & $\frac{b_{3} \tan \left(\gamma_{2}\right)-b_{2} \tan \left(\gamma_{3}\right)}{\tan \left(\gamma_{2}\right)-\tan \left(\gamma_{3}\right)}$ & $\frac{b_{1} \tan \left(\gamma_{3}\right)-b_{3} \tan \left(\gamma_{1}\right)}{\tan \left(\gamma_{3}\right)-\tan \left(\gamma_{1}\right)}$ & $\frac{b_{2} \tan \left(\gamma_{1}\right)-b_{1} \tan \left(\gamma_{2}\right)}{\tan \left(\gamma_{1}\right)-\tan \left(\gamma_{2}\right)}$ \\
\hline \hline
\end{tabular}

sensitivity coefficients, and, two aggregate sensitivity indices are determined to analyze the sensitivity of the pose of the moving platform of a 3-R $\underline{P R}$ manipulator to variations in its geometric parameters. Then, the contours of these indices are plotted in $M_{1}$, $M_{2}, M_{3}$ and $M_{4}$ RDWs and the results are analyzed.

\subsection{Sensitivity coefficients}

From the closed-loop kinematic chains $O-A_{i}-C_{i}-P-O$, $i=1, \ldots, 3$ depicted in Fig. 1 , the position vector $\mathbf{p}$ of point $P$ can be expressed in $\mathcal{F}_{b}$ as follows,

$$
\mathbf{p}=\left[\begin{array}{c}
p_{x} \\
p_{y}
\end{array}\right]=\mathbf{a}_{i}+\left(\mathbf{c}_{i}-\mathbf{a}_{i}\right)+\left(\mathbf{p}-\mathbf{c}_{i}\right), \quad i=1, \ldots, 3
$$

$\mathbf{a}_{i}$ and $\mathbf{c}_{i}$ being the position vectors of points $A_{i}$ and $C_{i}$ expressed in $\mathcal{F}_{b}$. Equation (9) can also be written as,

$$
\mathbf{p}=a_{i} \mathbf{h}_{i}+\rho_{i} \mathbf{u}_{i}+c_{i} \mathbf{k}_{i}
$$

with

$$
\mathbf{h}_{i}=\left[\begin{array}{c}
\cos \alpha_{i} \\
\sin \alpha_{i}
\end{array}\right], \mathbf{u}_{i}=\left[\begin{array}{c}
\cos \theta_{i} \\
\sin \theta_{i}
\end{array}\right], \mathbf{k}_{i}=\left[\begin{array}{c}
\cos \left(\phi+\beta_{i}+\pi\right) \\
\sin \left(\phi+\beta_{i}+\pi\right)
\end{array}\right]
$$

where $a_{i}$ is the distance between points $O$ and $A_{i}, \rho_{i}$ is the distance between points $A_{i}$ and $C_{i}, c_{i}$ is the distance between points $C_{i}$ and $P, \mathbf{h}_{i}$ is the unit vector $\overrightarrow{O A}_{i} /\left\|\overrightarrow{O A}_{i}\right\|_{2}, \mathbf{u}_{i}$ is the unit vector $\overrightarrow{A_{i} C_{i}} /\left\|\overrightarrow{A_{i} C_{i}}\right\|_{2}$ and $\mathbf{k}_{i}$ is the unit vector $\overrightarrow{C_{i} P} /\left\|\overrightarrow{C_{i} P}\right\|_{2}$.

Upon differentiation of Eq.(10), we obtain:

$$
\begin{aligned}
\delta \mathbf{p}= & \delta a_{i} \mathbf{h}_{i}+a_{i} \delta \alpha_{i} \mathbf{E} \mathbf{h}_{i}+\delta \rho_{i} \mathbf{u}_{i}+\rho_{i} \delta \theta_{i} \mathbf{E} \mathbf{u}_{i} \\
& +\delta c_{i} \mathbf{k}_{i}+c_{i}\left(\delta \phi+\delta \beta_{i}\right) \mathbf{E} \mathbf{k}_{i}
\end{aligned}
$$

with matrix $\mathbf{E}$ defined as

$$
\mathbf{E}=\left[\begin{array}{cc}
0 & -1 \\
1 & 0
\end{array}\right]
$$

$\delta \mathbf{p}$ and $\delta \phi$ being the position and orientation errors of the MP. Likewise, $\delta a_{i}, \delta \alpha_{i}, \delta \rho_{i}, \delta c_{i}$ and $\delta \beta_{i}$ denote the variations in $a_{i}, \alpha_{i}$, $\rho_{i}, c_{i}$ and $\beta_{i}$, respectively.

The idle variation $\delta \theta_{i}$ is eliminated by dot-multiplying Eq.(11) by $\rho_{i} \mathbf{u}_{i}^{T}$, thus obtaining

$$
\begin{aligned}
\rho_{i} \mathbf{u}_{i}^{T} \delta \mathbf{p}= & \rho_{i} \delta a_{i} \mathbf{u}_{i}^{T} \mathbf{h}_{i}+\rho_{i} a_{i} \delta \alpha_{i} \mathbf{u}_{i}^{T} \mathbf{E} \mathbf{h}_{i}+\rho_{i} \delta \rho_{i} \\
& +\rho_{i} \delta c_{i} \mathbf{u}_{i}^{T} \mathbf{k}_{i}+\rho_{i} c_{i}\left(\delta \phi+\delta \beta_{i}\right) \mathbf{u}_{i}^{T} \mathbf{E} \mathbf{k}_{i}
\end{aligned}
$$

Equation (13) can now be cast in vector form, namely,

$$
\begin{aligned}
& \mathbf{A}\left[\begin{array}{c}
\delta \phi \\
\delta \mathbf{p}
\end{array}\right]= \mathbf{H}_{a}\left[\begin{array}{l}
\delta a_{1} \\
\delta a_{2} \\
\delta a_{3}
\end{array}\right]+\mathbf{H}_{\alpha}\left[\begin{array}{l}
\delta \alpha_{1} \\
\delta \alpha_{2} \\
\delta \alpha_{3}
\end{array}\right]+\mathbf{B}\left[\begin{array}{l}
\delta \rho_{1} \\
\delta \rho_{2} \\
\delta \rho_{3}
\end{array}\right]+ \\
& \mathbf{H}_{c}\left[\begin{array}{l}
\delta c_{1} \\
\delta c_{2} \\
\delta c_{3}
\end{array}\right]+\mathbf{H}_{\beta}\left[\begin{array}{l}
\delta \beta_{1} \\
\delta \beta_{2} \\
\delta \beta_{3}
\end{array}\right]
\end{aligned}
$$

with

$$
\begin{aligned}
\mathbf{A} & =\left[\begin{array}{cc}
m_{1} & \rho_{1} \mathbf{u}_{1}^{T} \\
m_{2} & \rho_{2} \mathbf{u}_{2}^{T} \\
m_{3} & \rho_{3} \mathbf{u}_{3}^{T}
\end{array}\right], \mathbf{B}=\left[\begin{array}{ccc}
\rho_{1} & 0 & 0 \\
0 & \rho_{2} & 0 \\
0 & 0 & \rho_{3}
\end{array}\right] \\
\mathbf{H}_{a} & =\operatorname{diag}\left[\rho_{1} \mathbf{u}_{1}^{T} \mathbf{h}_{1} \rho_{2} \mathbf{u}_{2}^{T} \mathbf{h}_{2} \rho_{3} \mathbf{u}_{3}^{T} \mathbf{h}_{3}\right] \\
\mathbf{H}_{\alpha} & =\operatorname{diag}\left[\rho_{1} a_{1} \mathbf{u}_{1}^{T} \mathbf{E h}_{1} \rho_{2} a_{2} \mathbf{u}_{2}^{T} \mathbf{E h}_{2} \rho_{3} a_{3} \mathbf{u}_{3}^{T} \mathbf{E h}_{3}\right] \\
\mathbf{H}_{c} & =\operatorname{diag}\left[\rho_{1} \mathbf{u}_{1}^{T} \mathbf{k}_{1} \rho_{2} \mathbf{u}_{2}^{T} \mathbf{k}_{2} \rho_{3} \mathbf{u}_{3}^{T} \mathbf{k}_{3}\right] \\
\mathbf{H}_{\beta} & =\operatorname{diag}\left[\rho_{1} c_{1} \mathbf{u}_{1}^{T} \mathbf{E k}_{1} \rho_{2} c_{2} \mathbf{u}_{2}^{T} \mathbf{E} \mathbf{k}_{2} \rho_{3} c_{3} \mathbf{u}_{3}^{T} \mathbf{E k}_{3}\right]
\end{aligned}
$$

and

$$
m_{i}=-\rho_{i} c_{i} \mathbf{u}_{i}^{T} \mathbf{E} \mathbf{k}_{i}, \quad i=1, \ldots, 3
$$

Let us notice that $\mathbf{A}$ and $\mathbf{B}$ are the direct and the inverse Jacobian matrices of the manipulator, respectively. Assuming that $\mathbf{A}$ is non singular, i.e., the manipulator does not meet any Type II sin- 
gularity [26], we obtain upon multiplication of Eq.(14) by $\mathbf{A}^{-1}$ :

$$
\begin{aligned}
& {\left[\begin{array}{l}
\delta \phi \\
\delta \mathbf{p}
\end{array}\right]=} \mathbf{J}_{a}\left[\begin{array}{l}
\delta a_{1} \\
\delta a_{2} \\
\delta a_{3}
\end{array}\right]+\mathbf{J}_{\alpha}\left[\begin{array}{l}
\delta \alpha_{1} \\
\delta \alpha_{2} \\
\delta \alpha_{3}
\end{array}\right]+\mathbf{J}\left[\begin{array}{l}
\delta \rho_{1} \\
\delta \rho_{2} \\
\delta \rho_{3}
\end{array}\right]+ \\
& \mathbf{J}_{c}\left[\begin{array}{l}
\delta c_{1} \\
\delta c_{2} \\
\delta c_{3}
\end{array}\right]+\mathbf{J}_{\beta}\left[\begin{array}{l}
\delta \beta_{1} \\
\delta \beta_{2} \\
\delta \beta_{3}
\end{array}\right]
\end{aligned}
$$

with

$$
\begin{aligned}
\mathbf{J} & =\mathbf{A}^{-1} \mathbf{B} \\
\mathbf{J}_{a} & =\mathbf{A}^{-1} \mathbf{H}_{a} \\
\mathbf{J}_{\alpha} & =\mathbf{A}^{-1} \mathbf{H}_{\alpha} \\
\mathbf{J}_{c} & =\mathbf{A}^{-1} \mathbf{H}_{c} \\
\mathbf{J}_{\beta} & =\mathbf{A}^{-1} \mathbf{H}_{\beta}
\end{aligned}
$$

and

$$
\begin{aligned}
\mathbf{A}^{-1} & =\frac{1}{\operatorname{det}(\mathbf{A})}\left[\begin{array}{lll}
v_{1} & v_{2} & v_{3} \\
\mathbf{v}_{1} & \mathbf{v}_{2} & \mathbf{v}_{3}
\end{array}\right] \\
v_{i} & =\rho_{j} \rho_{k}\left(\mathbf{u}_{j} \times \mathbf{u}_{k}\right)^{T} \mathbf{k} \\
\mathbf{v}_{i} & =\mathbf{E}\left(m_{j} \rho_{k} \mathbf{u}_{k}-m_{k} \rho_{j} \mathbf{u}_{j}\right) \\
\operatorname{det}(\mathbf{A}) & =\sum_{i=1}^{3} m_{i} v_{i} \\
\mathbf{k} & =\mathbf{i} \times \mathbf{j}
\end{aligned}
$$

$j=(i+1)$ modulo $3 ; k=(i+2)$ modulo $3 ; i=1,2,3 . \mathbf{J}$ is the kinematic Jacobian matrix of the manipulator whereas $\mathbf{J}_{a}, \mathbf{J}_{\alpha}$ $\mathbf{J}_{c}$ and $\mathbf{J}_{\beta}$ are named sensitivity Jacobian matrices of the pose of the MP to variations in $a_{i}, \alpha_{i}, c_{i}$ and $\beta_{i}$, respectively. Indeed, the terms of $\mathbf{J}_{a}, \mathbf{J}_{\alpha}, \mathbf{J}_{c}$ and $\mathbf{J}_{\beta}$ are the sensitivity coefficients of the position and the orientation of the moving platform of the manipulator to variations in the Polar coordinates of points $A_{i}$ and $C_{i}$. Likewise, $\mathbf{J}$ contains the sensitivity coefficients of the pose of the MP of the manipulator to variations in the prismatic actuated joints. It is noteworthy that all these sensitivity coefficients are expressed algebraically.

Let $\delta a_{i x}$ and $\delta a_{i y}$ denote the position errors of points $A_{i}$, $i=1,2,3$, along $\overrightarrow{O x}$ and $\overrightarrow{O y}$, namely, the variations in the Cartesian coordinates of points $A_{i}$. Likewise, let $\delta c_{i X}$ and $\delta c_{i Y}$ denote the position errors of points $C_{i}$ along $\overrightarrow{P X}$ and $\overrightarrow{P Y}$, namely, the variations in the Cartesian coordinates of points $C_{i}$.
From Fig. 1,

$$
\begin{aligned}
& {\left[\begin{array}{l}
\delta a_{i x} \\
\delta a_{i y}
\end{array}\right]=\left[\begin{array}{cc}
\cos \alpha_{i} & -a_{i} \sin \alpha_{i} \\
\sin \alpha_{i} & a_{i} \cos \alpha_{i}
\end{array}\right]\left[\begin{array}{c}
\delta a_{i} \\
\delta \alpha_{i}
\end{array}\right]} \\
& {\left[\begin{array}{l}
\delta c_{i X} \\
\delta c_{i Y}
\end{array}\right]=\left[\begin{array}{cc}
\cos \beta_{i} & -c_{i} \sin \beta_{i} \\
\sin \beta_{i} & c_{i} \cos \beta_{i}
\end{array}\right]\left[\begin{array}{c}
\delta c_{i} \\
\delta \beta_{i}
\end{array}\right]}
\end{aligned}
$$

Accordingly, from Eq.(17) and Eqs.(20a)-(b), we obtain the following relation between the pose error of the MP and variations in the Cartesian coordinates of points $A_{i}$ and $C_{i}$ :

$$
\left[\begin{array}{l}
\delta \phi \\
\delta \mathbf{p}
\end{array}\right]=\mathbf{J}_{A}\left[\begin{array}{l}
\delta a_{1 x} \\
\delta a_{1 y} \\
\delta a_{2 x} \\
\delta a_{2 y} \\
\delta a_{3 x} \\
\delta a_{3 y}
\end{array}\right]+\mathbf{J}\left[\begin{array}{l}
\delta \rho_{1} \\
\delta \rho_{2} \\
\delta \rho_{3}
\end{array}\right]+\mathbf{J}_{C}\left[\begin{array}{l}
\delta c_{1 X} \\
\delta c_{1 Y} \\
\delta c_{2 X} \\
\delta c_{2 Y} \\
\delta c_{3 X} \\
\delta c_{3 Y}
\end{array}\right]
$$

$\mathbf{J}_{A}$ and $\mathbf{J}_{C}$ being named sensitivity Jacobian matrices of the pose of the MP to variations in the Cartesian coordinates of points $A_{i}$ and $C_{i}$, respectively. Indeed, the terms of $\mathbf{J}_{A}$ and $\mathbf{J}_{C}$ are the sensitivity coefficients of the pose of the MP to variations in the Cartesian coordinates of points $A_{i}$ and $C_{i}$.

In order to better highlight the sensitivity coefficients, let us write the $3 \times 6$ matrices $\mathbf{J}_{A}$ and $\mathbf{J}_{C}$ and the $3 \times 3$ matrix $\mathbf{J}$ as follows:

$$
\begin{aligned}
& \mathbf{J}_{A}=\left[\begin{array}{lll}
\mathbf{J}_{A_{1}} & \mathbf{J}_{A_{2}} & \mathbf{J}_{A_{3}}
\end{array}\right] \\
& \mathbf{J}_{C}=\left[\begin{array}{lll}
\mathbf{J}_{C_{1}} & \mathbf{J}_{C_{2}} & \mathbf{J}_{C_{3}}
\end{array}\right] \\
& \mathbf{J}=\left[\begin{array}{lll}
\mathbf{j}_{1} & \mathbf{j}_{2} & \mathbf{j}_{3}
\end{array}\right]
\end{aligned}
$$

the $3 \times 2$ matrices $\mathbf{J}_{A_{i}}$ and $\mathbf{J}_{C_{i}}$ and the three dimensional vectors $\mathbf{j}_{i}$ being expressed as:

$$
\begin{aligned}
\mathbf{J}_{A_{i}} & =\left[\begin{array}{l}
\mathbf{j}_{A_{i} \phi} \\
\mathbf{J}_{A_{i} p}
\end{array}\right], \quad i=1,2,3 \\
\mathbf{J}_{C_{i}} & =\left[\begin{array}{l}
\mathbf{j}_{C_{i} \phi} \\
\mathbf{J}_{C_{i} p}
\end{array}\right], \quad i=1,2,3 \\
\mathbf{j}_{i} & =\left[\begin{array}{l}
j_{i \phi} \\
\mathbf{j}_{i p}
\end{array}\right], \quad i=1,2,3
\end{aligned}
$$




$$
\begin{aligned}
& \mathbf{j}_{A_{i} \phi}=\frac{1}{\operatorname{det}(\mathbf{A})}\left[v_{i} q_{i} v_{i} r_{i}\right] \\
& \mathbf{j}_{C_{i} \phi}=\frac{1}{\operatorname{det}(\mathbf{A})}\left[v_{i} s_{i} v_{i} t_{i}\right] \\
& j_{i \phi}=\frac{\rho_{i} v_{i}}{\operatorname{det}(\mathbf{A})} \\
& \mathbf{J}_{A_{i} p}=\frac{1}{\operatorname{det}(\mathbf{A})}\left[\begin{array}{cc}
q_{i} \mathbf{v}_{i}^{T} \mathbf{i} & r_{i} \mathbf{v}_{i}^{T} \mathbf{i} \\
q_{i} \mathbf{v}_{i}^{T} \mathbf{j} & r_{i} \mathbf{v}_{i}^{T} \mathbf{j}
\end{array}\right] \\
& \mathbf{J}_{C_{i} p}=\frac{1}{\operatorname{det}(\mathbf{A})}\left[\begin{array}{c}
s_{i} \mathbf{v}_{i}^{T} \mathbf{i} t_{i} \mathbf{v}_{i}^{T} \mathbf{i} \\
s_{i} \mathbf{v}_{i}^{T} \mathbf{j} t_{i} \mathbf{v}_{i}^{T} \mathbf{j}
\end{array}\right] \\
& \mathbf{j}_{i p}=\frac{1}{\operatorname{det}(\mathbf{A})}\left[\begin{array}{c}
\rho_{i} \mathbf{v}_{i}^{T} \mathbf{i} \\
\rho_{i} \mathbf{v}_{i}^{T} \mathbf{j}
\end{array}\right]
\end{aligned}
$$

$q_{i}, r_{i}, s_{i}$ and $t_{i}$ taking the form:

$$
\begin{aligned}
q_{i} & =\rho_{i} \mathbf{u}_{i}^{T} \mathbf{i} \\
r_{i} & =\rho_{i} \mathbf{u}_{i}^{T} \mathbf{j} \\
s_{i} & =\rho_{i} \mathbf{u}_{i}^{T} \mathbf{k}_{i} \cos \beta_{i}-\rho_{i} \mathbf{u}_{i}^{T} \mathbf{E} \mathbf{k}_{i} \sin \beta_{i} \\
t_{i} & =\rho_{i} \mathbf{u}_{i}^{T} \mathbf{k}_{i} \sin \beta_{i}+\rho_{i} \mathbf{u}_{i}^{T} \mathbf{E} \mathbf{k}_{i} \cos \beta_{i}
\end{aligned}
$$

$\mathbf{j}_{A_{i} \phi}, \mathbf{j}_{C_{i} \phi}$ and $j_{i \phi}$ contain the sensitivity coefficients of the orientation of the MP of the manipulator to variations in the Cartesian coordinates of points $A_{i}, C_{i}$ and prismatic actuated variables, respectively. Similarly, $\mathbf{J}_{A_{i} p}, \mathbf{J}_{C_{i} p}$ and $\mathbf{j}_{i p}$ contain the sensitivity coefficients related to the position of the MP.

Accordingly, the designer of such a planar parallel manipulator can easily identify the most influential geometric variations to the pose of its MP and synthesize proper dimensional tolerances from the previous sensitivity coefficients. Two aggregate sensitivity indices related to the geometric errors of the moving and base platforms are introduced thereafter.

\subsection{Global sensitivity indices}

The pose errors of the manipulator MP depend on variations in the geometric parameters as well as on the manipulator configuration. In order to analyze the influence of the manipulator configuration on those errors, let us first formulate some indices in order to assess the aggregate sensitivity of the MP pose to variations in the geometric parameters for a given manipulator configuration. To this end, let Eq.(21) be expressed as:

$$
\left[\begin{array}{c}
\delta \phi \\
\delta \mathbf{p}
\end{array}\right]=\mathbf{J}_{s}\left[\delta \mathbf{a}_{\mathbf{i}} \delta \rho_{\mathbf{i}} \delta \mathbf{c}_{\mathbf{i}}\right]^{T}
$$

$$
\mathbf{J}_{s}=\left[\mathbf{J}_{A} \mathbf{J} \mathbf{J}_{C}\right]
$$

and

$$
\begin{aligned}
& \delta \mathbf{a}_{\mathbf{i}}=\left[\delta a_{1 x} \delta a_{1 y} \delta a_{2 x} \delta a_{2 y} \delta a_{3 x} \delta a_{3 y}\right] \\
& \delta \rho_{\mathbf{i}}=\left[\begin{array}{lll}
\delta \rho_{1} & \delta \rho_{2} & \delta \rho_{3}
\end{array}\right]
\end{aligned}
$$

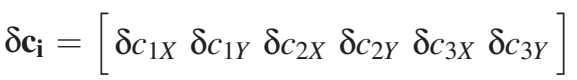

The $3 \times 15$ matrix $\mathbf{J}_{s}$ is named "sensitivity Jacobian matrix" and can be written as follows:

$$
\mathbf{J}_{s}=\left[\begin{array}{l}
\mathbf{j}_{s_{\phi}} \\
\mathbf{J}_{s_{p}}
\end{array}\right]
$$

with

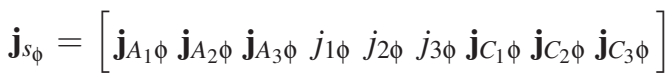

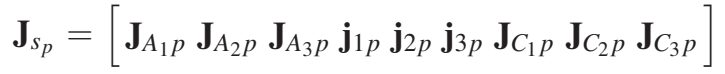

From Eq.(30a), we can define an aggregate sensitivity index $v_{\phi}$ of the orientation of the MP of the manipulator to variations in its geometric parameters and prismatic actuated joints, namely,

$$
v_{\phi}=\frac{\left\|\mathbf{j}_{s_{\phi}}\right\|_{2}}{n_{v}}
$$

$n_{v}$ being the number of variations that are considered. Here, $n_{v}$ is equal to 15 .

Likewise, from Eq.(30b), an aggregate sensitivity index $v_{p}$ of the position of the MP of the manipulator to variations in its geometric parameters and prismatic actuated joints can be defined as follows:

$$
v_{p}=\frac{\left\|\mathbf{J}_{s_{p}}\right\|_{2}}{n_{v}}
$$

For any given manipulator configuration, the lower $v_{\phi}$, the lower the overall sensitivity of the orientation of its MP to variations in the geometric parameters. Similarly, the lower $v_{p}$, the 
lower the overall sensitivity of the MP position to variations in the geometric parameters. As a matter of fact, $v_{\phi}\left(v_{p}\right.$, resp.) characterizes the intrinsic sensitivity of the MP orientation (position, resp.) to any variation in the geometric parameters. Let us notice that $v_{p}$ as well as the sensitivity coefficients related to the MP position defined in Sections 5.1 are frame dependent, whereas $v_{\phi}$ and the sensitivity coefficients related to the MP orientation are not.

In order to evaluate the sensitivity of the manipulator over its workspace or part of it, four global sensitivity indices are defined as follows:

$$
\begin{aligned}
\overline{v_{\phi}} & =\frac{\int_{W} v_{\phi} \mathrm{d} W}{\int_{W} \mathrm{~d} W} \\
v_{\phi_{\max }} & =\max \left(\mathrm{v}_{\phi}\right) \\
\overline{v_{p}} & =\frac{\int_{W} v_{p} \mathrm{~d} W}{\int_{W} \mathrm{~d} W} \\
v_{p_{\max }} & =\max \left(\mathrm{v}_{p}\right)
\end{aligned}
$$

$\overline{v_{\phi}}$ and $\overline{v_{p}}$ are the average values of $v_{\phi}$ and $v_{p}$ over $W, W$ being the manipulator workspace or part of it. Likewise, $v_{\phi_{\max }}$ and $v_{p_{\max }}$ are the maximum values of $v_{\phi}$ and $v_{p}$ over $W$.

Finally, $v_{\phi}, \overline{v_{\phi}}$ and $v_{\phi_{\max }}$ are expressed in $[\mathrm{rad} / \mathrm{L}]$, whereas $\mathrm{v}_{p}$, $\overline{v_{p}}$ and $v_{p_{\max }}$ are dimensionless, [L] being the unit of length.

\subsection{Comparison of two non-degenerate and two de- generate 3-RPR PPMs}

In this section, the sensitivity of $M_{1}, M_{2}, M_{3}$ and $M_{4}$ is evaluated within their RDW for a matter of comparison based on aggregate sensitivity indices $v_{\phi}$ and $v_{p}$ defined in Eqs.(31) and (32) and global sensitivity indices $\overline{v_{\phi}}, v_{\phi_{\max }}, \overline{v_{p}}$ and $v_{p_{\max }}$ defined in Eqs.(33a)-(d).

Figures 5(a)-(d) (Figures 6(a)-(d), resp.) illustrate the isocontours of the maximum value of $v_{\phi}\left(v_{p}\right.$, resp.). for a given orientation $\phi$ of the MP throughout the RDW of $M_{1}, M_{2}, M_{3}$ and $M_{4}$, respectively.

Table 3.

\begin{tabular}{c|cccc}
\multicolumn{4}{c}{ Values of $\overline{\nabla_{\phi}}, v_{\phi_{\max }}, \overline{v_{p}}$ and $v_{p_{\max }}$ for $M_{1}, M_{2}, M$} \\
\hline \hline & $M_{1}$ & $M_{2}$ & $M_{3}$ & $M_{4}$ \\
\hline$\overline{v_{\phi}}$ & 0.292 & 0.254 & 0.233 & 0.192 \\
$v_{\phi_{\max }}$ & 0.426 & 0.365 & 0.386 & 0.322 \\
$\overline{v_{p}}$ & 0.171 & 0.231 & 0.194 & 0.316 \\
$v_{p_{\max }}$ & 0.263 & 0.327 & 0.284 & 0.441 \\
\hline \hline
\end{tabular}

Table 3 and Fig. 7 illustrate the values of $\overline{v_{\phi}}, v_{\phi_{\max }}, \overline{v_{p}}$ and $v_{p_{\max }}$ for the four manipulators under study. It is apparent that

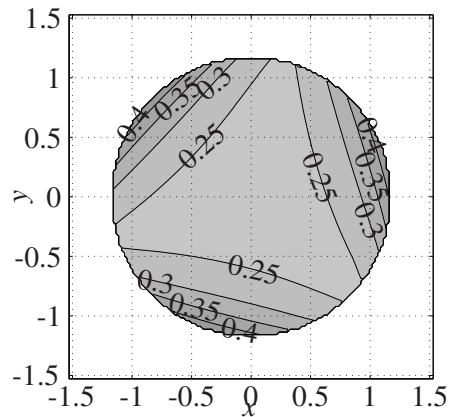

(a) $M_{1}: v_{\phi}$

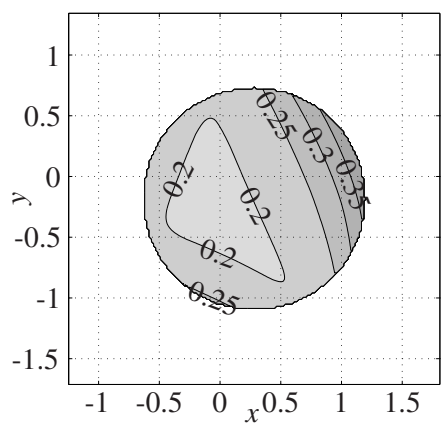

(c) $M_{3}: v_{\phi}$

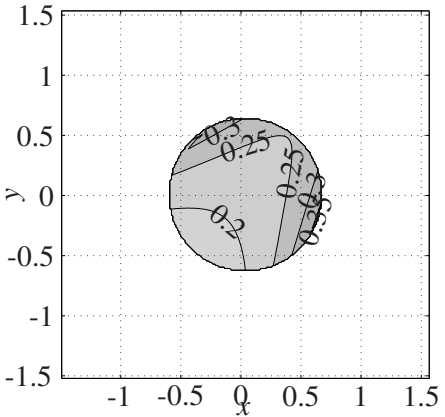

(b) $M_{2}: v_{\phi}$

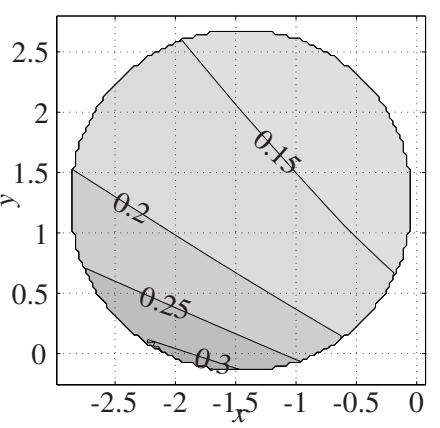

(d) $M_{4}: v_{\phi}$
Figure 5. $\quad v_{\phi}$ isocontours of: (a) $M_{1}$, (b) $M_{2}$, (c) $M_{3}$ and (d) $M_{4}$

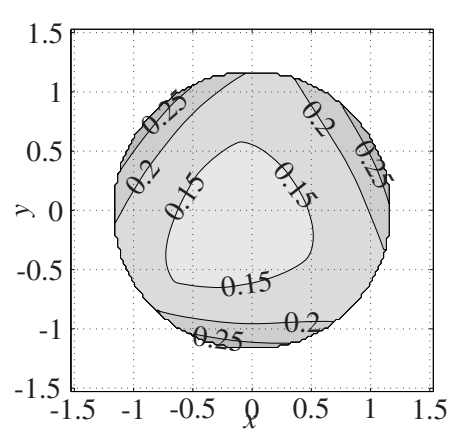

(a) $M_{1}: v_{p}$

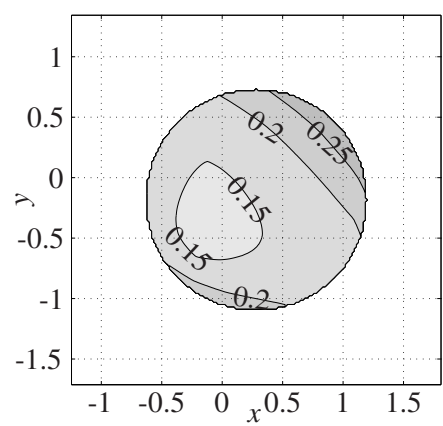

(c) $M_{3}: v_{p}$

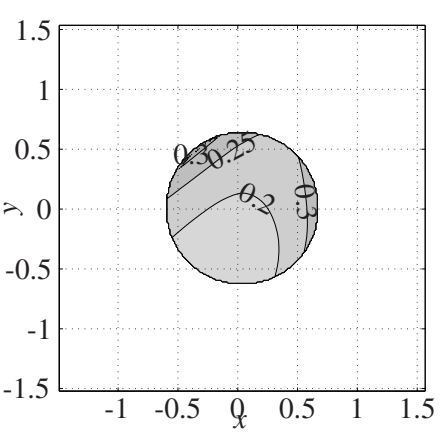

(b) $M_{2}: v_{p}$

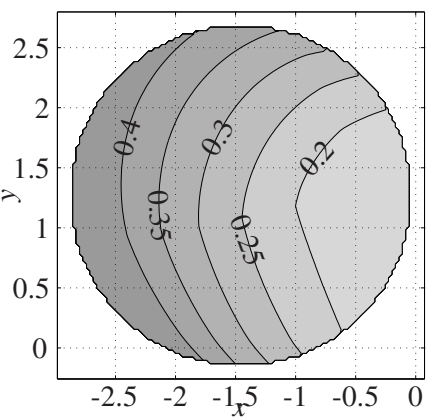

(d) $M_{4}: v_{p}$
Figure 6. $\quad v_{p}$ isocontours of: (a) $M_{1}$, (b) $M_{2}$, (c) $M_{3}$ and (d) $M_{4}$

$M_{4}$ has the least sensitive orientation of its MP and that $M_{1}$ has the least sensitive position of its MP. On the contrary, $M_{4}$ has the most sensitive position of its MP and $M_{1}$ has the most sensitive orientation of its MP. 


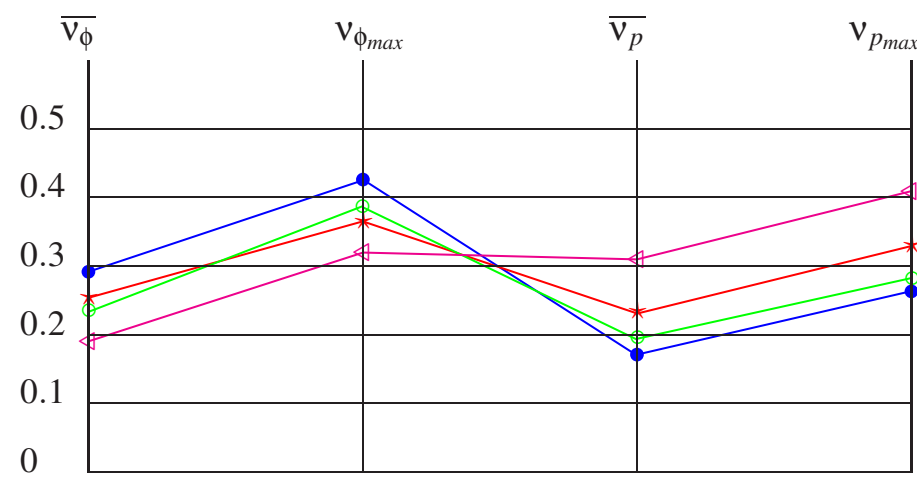

Figure 7. Comparison of $\bullet \bullet: M_{1}, \star-\star: M_{2}, \circ-\circ: M_{3}, \triangleleft-\triangleleft: M_{4}$

\section{Sensitivity Comparison of Two Actuating Modes}

In this section, two actuating modes of the 3-RPR PPM, namely the 3-RPR PPM and the 3-Rㅛ R PPM, are compared with regard to their sensitivity to variations in geometric parameters ${ }^{2}$.

Table 4 shows the eight actuating modes of the 3-RPR PPM. For instance, the first actuating mode corresponds to the 3$\underline{R P R}$ PPM, also called $\underline{R} P R_{1}-\underline{R} P R_{2}-\underline{R} P R_{3}$ PPM in the scope of this paper, as the first revolute joints (located at points $A_{i}$ ) of its limbs are actuated. Likewise, the eighth actuating mode corresponds to the $3-R \underline{P} R$ PPM, also called $R \underline{P} R_{1}-R \underline{P} R_{2}-R \underline{P} R_{3}$ PPM, as the prismatic joints of its limbs are actuated. For the fourth actuating mode, the prismatic joint of the first limb is actuated while the first revolute joints of the two other limbs are actuated.

\begin{tabular}{c|c|c} 
Table 4. & The eight actuating modes of the 3-RPR P \\
\hline \hline \multicolumn{2}{|c|}{ Actuating mode number } & active angles \\
\hline 1 & $\underline{R} P R_{1}-\underline{R} P R_{2}-\underline{R} P R_{3}$ & $\theta_{1}, \theta_{2}, \theta_{3}$ \\
2 & $\underline{R} P R_{1}-\underline{R} P R_{2}-R \underline{P} R_{3}$ & $\theta_{1}, \theta_{2}, \rho_{3}$ \\
3 & $\underline{R} P R_{1}-R \underline{P} R_{2}-\underline{R} P R_{3}$ & $\theta_{1}, \rho_{2}, \theta_{3}$ \\
4 & $R \underline{P} R_{1}-\underline{R} P R_{2}-\underline{R} P R_{3}$ & $\rho_{1}, \theta_{2}, \theta_{3}$ \\
5 & $\underline{R} P R_{1}-R \underline{R} R_{2}-R \underline{P} R_{3}$ & $\theta_{1}, \rho_{2}, \rho_{3}$ \\
6 & $R \underline{P} R_{1}-R \underline{P} R_{2}-\underline{R} P R_{3}$ & $\rho_{1}, \rho_{2}, \theta_{3}$ \\
7 & $R \underline{P} R_{1}-\underline{R} P R_{2}-R \underline{P} R_{3}$ & $\rho_{1}, \theta_{2}, \rho_{3}$ \\
8 & $R \underline{P} R_{1}-R \underline{P} R_{2}-R \underline{P} R_{3}$ & $\rho_{1}, \rho_{2}, \rho_{3}$ \\
\hline \hline
\end{tabular}

Table 5. RDW radius of $M_{1}$ and $M_{5}$

\begin{tabular}{cc}
\hline \hline$R_{1}$ & $R_{5}$ \\
1.21 & 1.60 \\
\hline
\end{tabular}

\footnotetext{
${ }^{2}$ As the actuators are not of the same type for the two manipulators (revolute actuators for the first one and prismatic actuators of the second one), their variations are not considered in order the sensitivity comparison of the two manipulators to make sense.
}

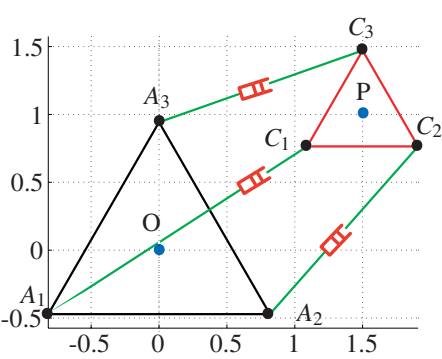

(a) $M_{1}$

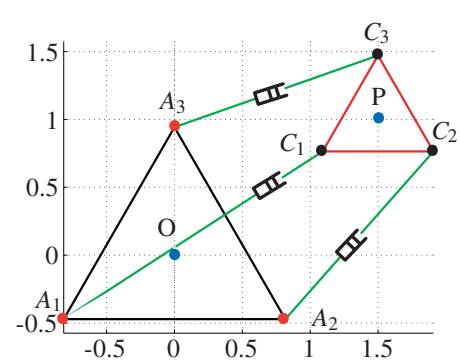

(b) $M_{5}$
Figure 8. Two actuating modes: (a) 3-R $\underline{P} R$ manipulator, (b) 3- $\underline{R} P R$ manipulator, $\phi=0$ and $\mathbf{p}=[1.5,1]^{T}$

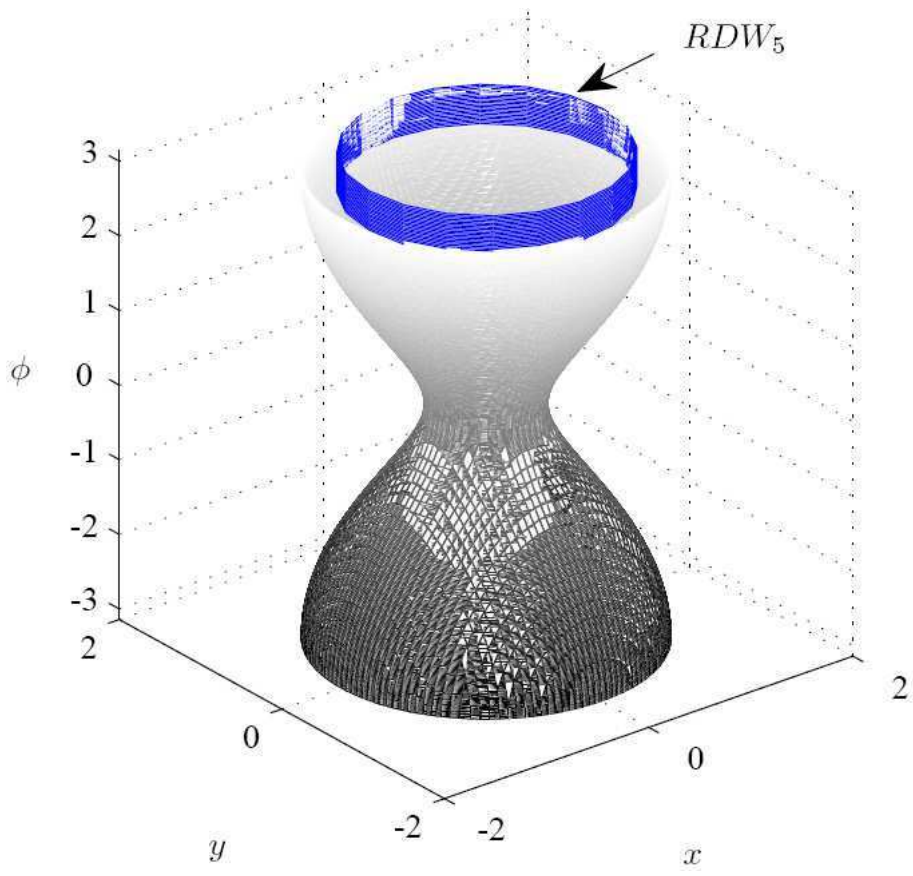

Figure 9. $M_{5}$ Regular Dexterous Workspace

Let $M_{1}$ and $M_{5}$ denote the 3-RPR and the 3-RPR PPMs, respectively, as shown in Figs. 8(a)-(b). The RDW of $M_{5}$ is illustrated in Fig. 9. From Table 5, we can notice that the RDW of $M_{5}$ is larger than the one of $M_{1}$.

Figures 10(a)-(b) show the isocontours of the maximum value of $v_{\phi M_{1}}$ and $v_{\phi M_{5}}$ throughout the RDW of $M_{1}$ and $M_{5}$. Likewise, Figs. 11(a)-(b) show the isocontours of the maximum value of $v_{p M_{1}}$ and $v_{p M_{5}}$. As a matter of fact, those isocontours correspond to the maximal global positioning and orientation errors with regard to the orientation $\phi$ of the moving platform of the manipulator.

Table 6 and Fig. 12 illustrate the values of $\overline{v_{\phi}}, v_{\phi_{\max }}, \overline{v_{p}}$ and $v_{p_{\max }}$ for the two actuating modes under study. It is apparent that $M_{1}$ is better than $M_{5}$, both in terms of orientation and positioning errors of its moving platform due to variations in geometric parameters. 


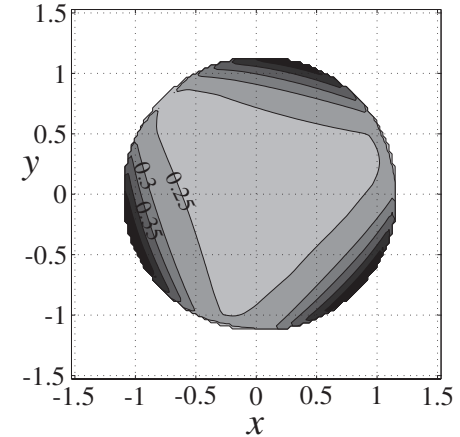

(a)

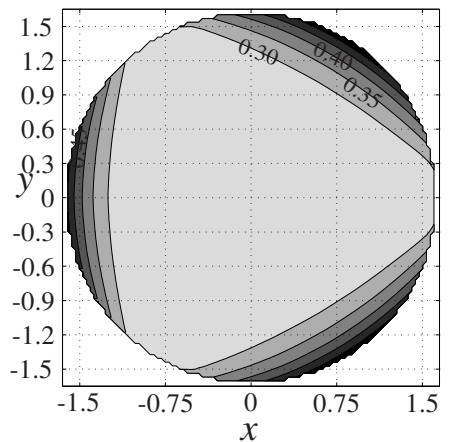

(b)
Figure 10. (a) $M_{1} \mathrm{v}_{\phi}$ and (b) $M_{5} \mathrm{v}_{\phi}$ isocontours

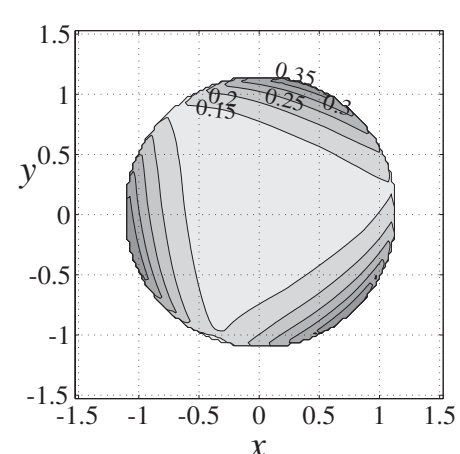

(a)

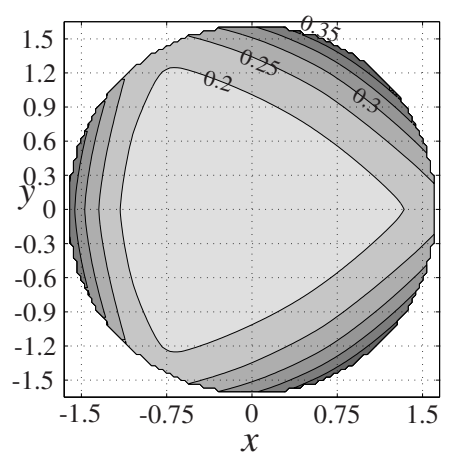

(b)
Figure 11. (a) $M_{1} \mathrm{v}_{p}$ and (b) $M_{5} \mathrm{v}_{p}$ isocontours

Table 6. Values of $\overline{v_{\phi}}, v_{\phi \max }, \overline{v_{p}}$ and $v_{\text {pmax }}$ for $M_{1}$ and $M_{5}$

\begin{tabular}{c|cc}
\hline \hline & $M_{1}$ & $M_{5}$ \\
\hline$\overline{v_{\phi}}$ & 0.251 & 0.289 \\
$v_{\phi_{\max }}$ & 0.448 & 0.501 \\
$\overline{v_{p}}$ & 0.163 & 0.222 \\
$v_{p_{\max }}$ & 0.369 & 0.423 \\
\hline \hline
\end{tabular}

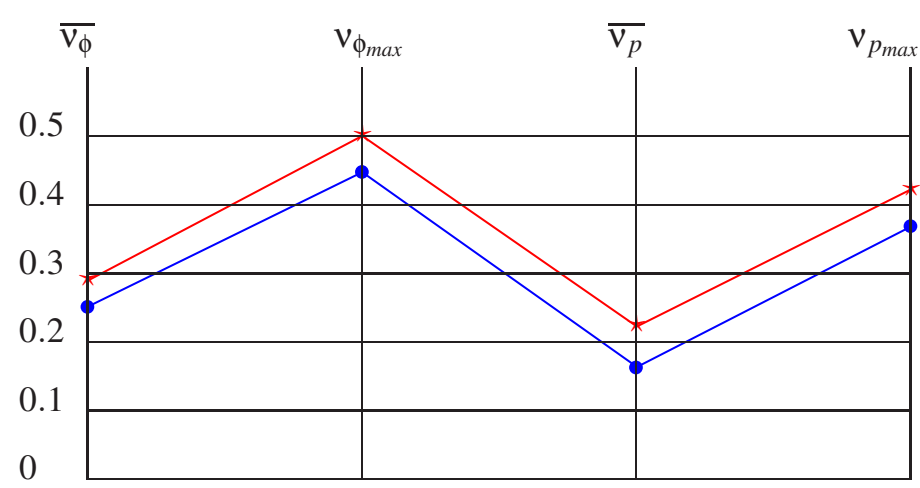

Figure 12. Comparison of $\bullet-\bullet: M_{1}$ and $\star-\star: ~ M 5$

\section{Conclusions}

This paper dealt with the sensitivity analysis of 3-RPR planar parallel manipulators (PPMs). First, the manipulators under study as well as their degeneracy conditions were presented. Then, an optimization problem was formulated in order to obtain their maximal regular dexterous workspace (RDW). Accordingly, the sensitivity of the pose of their moving platform to variations in geometric parameters was evaluated within their RDW. Then, a methodology was proposed to compare PPMs with regard to their dexterity and sensitivity. Four 3-RPR PPMs were compared as illustrative examples. Moreover, two actuating modes were compared with regard to their sensitivity to geometric uncertainties. Finally, four global sensitivity indices were introduced in order to evaluate the sensitivity of PPMs over their Cartesian workspace. Those indices characterize the intrinsic sensitivity of the moving platform pose to any variation in the geometric parameters. They are like amplification factors of errors in geometric parameters. There values remain always lower than one for the manipulators under study. It means that there is no amplification of errors in geometric parameters. The proposed indices can also be used to help the designer of PPMs select a good manipulator architecture at the conceptual design stage.

\section{REFERENCES}

[1] Wang, J., and Masory, O., (1993). "On the accuracy of a Stewart platform - Part I, The effect of manufacturing tolerances," In: Proceedings of the IEEE International Conference on Robotics Automation, ICRA'93, Atlanta, USA, pp. 114-120.

[2] Kim, H.S. and Choi, Y.J. (2000). "The Kinematic Error Bound Analysis of the Stewart Platform," Journal of Robotic Systems, 17, pp. 63-73.

[3] Kim, H.S., and Tsai, L-W., (2003). "Design optimization of a Cartesian parallel manipulator," ASME Journal of Mechanical Design, 125, pp. 43-51.

[4] Caro, S., Bennis, F., and Wenger, P., (2005). "Tolerance Synthesis of Mechanisms: A Robust Design Approach,” ASME Journal of Mechanical Design, 127, pp. 86-94.

[5] Cardou, P., Bouchard, S., and Gosselin, C. (2010). "Kinematic-sensitivity indices for dimensionally nonhomogeneous jacobian matrices," IEEE Transactions on Robotics, 26(1), pp. 166-173.

[6] Caro, S., Wenger, P., Bennis, F., and Chablat, D., (2006). "Sensitivity Analysis of the Orthoglide, A 3-DOF Translational Parallel Kinematic Machine," ASME Journal of Mechanical Design, 128, March, pp. 392-402.

[7] Yu, A., Bonev, I.A. and Zsombor-Murray, P.J. (2007). "Geometric Method for the Accuracy Analysis of a Class of 3DOF Planar Parallel Robots," Mechanism and Machine Theory, 43(3), pp. 364-375.

[8] Meng, J., Zhang, D. and Li, Z. (2009). Accuracy Analysis of Parallel Manipulators With Joint Clearance, ASME Journal of Mechanical Design, 131, January, pp. 011013-1-011013- 
9.

[9] Binaud, N., Caro, S. and Wenger, P. (2010). "Sensitivity Comparison of Planar Parallel Manipulators," Mechanism and Machine Theory, 45, pp. 1477-1490.

[10] Hunt K.H., (1978). Kinematic Geometry of Mechanisms, Oxford University Press, Cambridge.

[11] Hunt K.H., (1983). "Structural kinematics of in-parallel actuated robot arms," J. of Mechanisms, Transmissions and Automation in Design, 105(4), pp.705-712.

[12] Gosselin C., Sefrioui J. and Richard M. J., (1992). "Solutions polynomiales au problème de la cinématique des manipulateurs parallèles plans trois degrés de liberté," Mechanism and Machine Theory, 27, pp. 107-119.

[13] Pennock G.R. and Kassner D.J., (1990). "Kinematic analysis of a planar eight-bar linkage: application to a platformtype robot," ASME Proc. of the 21th Biennial Mechanisms Conf., Chicago, pp. 37-43.

[14] Gosselin C.M. and Merlet J-P, (1994). "On the direct kinematics of planar parallel manipulators: special architectures and number of solutions," Mechanism and Machine Theory, 29(8), pp. 1083-1097.

[15] Kong X. and Gosselin C.M., (2001). "Forward displacement analysis of third-class analytic 3-RPR planar parallel manipulators," Mechanism and Machine Theory, 36, pp.10091018.

[16] Wenger P., Chablat D., Zein M., (2007). “Degeneracy study of the forward kinematics of planar 3-RPR parallel manipulators," ASME Journal of Mechanical Design, 129, pp. 1265-1268.

[17] Liu, X.-J., Wang, J. and Pritschow, G. (2006). "Kinematics, Singularity and Workspace of Planar 5R Symmetrical Parallel Mechanisms," Mechanism and Machine Theory, 41(2), pp. 145-169.

[18] Liu, X.-J., Wang, J. and Pritschow, G. (2006). "Performance Atlases and Optimum Design of Planar 5R Symmetrical Parallel Mechanisms," Mechanism and Machine Theory, 41(2), pp. 119-144.

[19] Liu, X.-J., Wang, J. and Pritschow, G. (2006). “On the Optimal Design of the PRRRP 2-DOF Parallel Mechanism," Mechanism and Machine Theory, 41(9), pp. 1111-1130.

[20] Caro, S., Chablat, D., Wenger, P. and Angeles, J. (2003). "Isoconditioning Loci of Planar Three-Dof Parallel Manipulators," In: G. Gogu, D. Coutellier, P. Chedmail and P. Ray (Editors), Recent Advances in Integrated Design and Manufacturing in Mechanical Engineering, Kluwer Academic Publisher, 2003, pp. 129-138.

[21] Caro, S., Binaud, N. and Wenger, P. (2009). "Sensitivity Analysis of 3-RPR Planar Parallel Manipulators," ASME Journal of Mechanical Design, 131, pp. 121005-1121005-
13.

[22] Rakotomanga, N., Chablat, D. and Caro, S., (2008). Kinetostatic Performance of a Planar Parallel Mechanism with Variable Actuation, Advances in Robot Kinematics, pp. 311-320.

[23] Arakelian, V., Briot, S. and Glazunov, V. (2008). "Increase of Singularity-Free Zones in the Workspace of Parallel Manipulators Using Mechanisms of Variable Structure,” Mechanism and Machine Theory, 43(9), pp. 1129-1140.

[24] Ranjbaran, F., Angeles, J., Gonzalez-Palacios, M. A. and Patel, R. V. (1995), The Mechanical Design of a SevenAxes Manipulator with Kinematic Isotropy, ASME Journal of Intelligent and Robotic Systems, vol. 14, no. 1, pp. 21-41.

[25] Al-Sultan, K.S. and Al-Fawzan, M.A. (1997). "A tabu search Hook and Jeeves algorithm for unconstrained optimization," European journal of operational research, 103, pp. 198-208.

[26] Gosselin, C.M. and Angeles, J. (1990). "Singularity Analysis of Closed-Loop Kinematic Chains," IEEE Transactions on Robotics and Automatics, 6(3), pp. 281-290. 